\title{
A new force field of formamide and the effect of the dielectric constant on miscibility
}

\author{
January 29, 2015
}

Alexander Pérez de la Luz ${ }^{\mathrm{a}}$, G. Arlette Méndez-Maldonado ${ }^{\mathrm{a}}$, Edgar Núñez-Rojas ${ }^{\mathrm{a}}$, Fernando Bresme ${ }^{\mathrm{b}}$ and José Alejandre ${ }^{\mathrm{a} *}$

a Departamento de Química, Universidad Autónoma Metropolitana-Iztapalapa. Av. San Rafael Atlixco 186, Col. Vicentina, 09340, México Distrito Federal, México;

${ }^{b}$ Department of Chemistry, Imperial College-London, SW7 2AZ, United Kingdom, and Department of Chemistry, Norwegian University of Science and Technology,

Trondheim, Norway

*Author to whom correspondence should be addressed. E-mail: jra@xanum.uam.mx 


\begin{abstract}
Current force fields underestimate significantly the dielectric constant of formamide at standard conditions. We present a derivation of an accurate potential for formamide, which functional form builds on the OPLS/AA force field. Our procedure follows the approach introduced by Salas et al. (J. Chem. Theory and Comp., Just accepted, 2015), that can use information from $a b$ initio calculations and molecular dynamics simulations. We consider several strategies to derive the atomic charges of formamide. We find that the inclusion of polarization effects in the quantum mechanical computations is essential to obtain reliable force fields. By varying the atomic charges and the LennardJones parameters describing the dispersion interactions in the OPLS/AA force field, we derive an optimum set of parameters to obtain accurate results for the dielectric constant, surface tension and bulk density of liquid formamide in a wide range of thermodynamic states. We test the transferability of our parameters to investigate liquid/liquid mixtures. We have chosen as case study an equimolar mixture of formamide and hexan-2-one. This mixture involves two fluids with very different polar characteristics, namely, large differences in their dielectric constants and their performance as polar solvents. The new potential highlights the importance of the correct parametrization of the pure liquid phases to investigate liquid mixtures. Finally, we examine the microscopic origin of the observed inmiscibility between formamide and hexa-2-one.
\end{abstract}




\section{Introduction}

Formamide is a highly polar molecule that forms strong hydrogen bonds through interactions between hydrogen and oxygen atoms lying in two different molecules (C$\mathrm{H} . . . \mathrm{O}$ and $\mathrm{N}-\mathrm{H} . . . \mathrm{O})$. The hydrogen bond character of the liquid makes formamide an excellent reference system to study the hydrophilic and hydrophobic interactions [1]. Further, formamide has attracted the attention of biologist and biochemists, since it is the smallest unit featuring in a peptidic chain. Also, formamide has been adopted as a model to investigate the formation and breaking of peptidic bonds[2]. The description of these bonds is essential to understand the physico-chemical properties of proteins.

A considerable effort has been devoted in the last thirty years to develop force fields capable of accurately reproducing the thermodynamic properties of complex fluids in a wide range of temperatures and pressures. Often, force fields have focused in reproducing the bulk density and heat of vaporization of liquids [3, 4, 5]. This approach does not warranty the accurate prediction of all the properties and indeed deviations between simulation and experiment, e.g. critical temperature and orthobaric densities, have been reported [6]. This has prompted the development of alternative parametrization strategies, targeting e.g. the critical temperature and liquid coexistence density $[7,8]$. However, very often these force fields fail to reproduce the dielectric constant of polar fluids [9]. One possible reason for this may be connected to the approach used to derive the atomic charges, which has relied on quantum mechanical computations of molecules in vacuum, hence neglecting molecular correlations.

The properties of pure formamide have been investigated in a number of computer simulation studies $[5,10]$. The latter approach takes into account multibody correlations, but requires the use of a force field to compute the properties of the condensed phase. One of these force fields is the Optimized Potential for Liquid Simulations (OPLS), which was originally derived for amides by Jorgensen and Swenson in 1985. [5] Later, in 1997, Essex and Jorgensen reported [11] the dielectric constant of formamide and dimethylformamide using isothermal isobaric Monte Carlo (MC) simulations with the OPLS force field.[5] The simulation result for formamide, 59, is about half of the measured dielectric constant, 109. Pohuvski et al. in 2003, [12] reported the structural and dynamic properties of formamide using an intermolecular potential derived from $a b$ initio computations. The force field was fitted to reproduce the structure obtained from neutron diffraction. Cordeiro [13] in 1997 performed MC simulations to derive a force field of formamide able to reproduce the experimental liquid density and heat of vaporization. Bako et al. published later on [14] an analysis of the hydrogen bond structure of formamide using the OPLS [5] and Cordeiro's [13] potentials. It has been suggested that the failure of current force fields to reproduce the dielectric constant of liquids is connected to the atomic charges that have been 
derived without considering intermolecular polarization effects, which would be relevant in condensed phases. One step in that direction are the computations of the dielectric properties of N-methyl-formamide and N-N methyl-formamide by Harder et al. in 2008, using polarizable force field based on the classical Drude oscillator [15]. The simulation results were close to the experimental data.

Recently, Caleman et al. reported [16] an extensive investigation containing force field benchmark data for 146 organic liquids. The accuracy of the OPLS/AA and GAFF force fields $[3,4]$ to reproduce the liquid density, enthalpy of vaporization, heat capacity, surface tension, isothermal compressibility, volumetric thermal expansion coefficient and dielectric constant was assessed. Most of the calculated properties agree well with the experimental results, but the surface tension and dielectric constant were systematically lower than the experiment. The deviations for surface tensions were $\sim 70 \%$ and $\sim 90 \%$ for GAFF and OPLS/AA, respectively. Zubillaga et al. [17] revisited the OPLS/AA force field computations using a longer truncation distance for the Lennard-Jones dispersion interactions. The new surface tension results were in much better agreement with the experiment, highlighting the importance of long range corrections in the computation of the pressure tensor. The dielectric constant calculated by Zubillaga et al. [17] was not sensitive to changes on truncation distance. Both force fields, GAFF and OPLS/AA, underestimated the experimental dielectric constant of most of the 146 organic liquids by $\sim 50 \%$. For the specific case of formamide the dielectric constant at $298.15 \mathrm{~K}$ and 1 bar was 41 and 50 using GAFF and OPLS/AA force fields, respectively.

From the discussion above one may conclude that it is difficult to obtain the correct dielectric constant of formamide without including explicitly polarization effects. However, it has been shown recently $[9,18,19]$ that it is possible to reproduce the experimental dielectric constant of water using non-polarizable force fields. Alejandre et al. developed the TIP4Q and TIP4P / $\epsilon$ non-polarizable water force fields $[18,19]$. The parameters were derived to reproduce simultaneously the liquid dielectric constant and the temperature of maximum density. Both models reproduced other thermodynamic and transport properties, including the water density anomalies. This work shows that rigid models can indeed reproduce the experimental data. We exploit this approach in this paper.

In a recent work, Salas et al. introduced [9] a procedure that enables the systematic derivation of a liquid force field, by fitting the atomic charges to reproduce the dielectric constant, and the Lennard-Jones (LJ) parameters, $\epsilon_{L J}$ and $\sigma_{L J}$, to reproduce the surface tension and liquid density, respectively. This procedure has been applied to methanol, pyridine, diclhoromethane and EMIM-BF4 liquids. The resulting force fields represent an improvement over existing ones, providing generally a better representation of the experimental data. We note that the molecules investi- 
gated with that procedure do not have very high dipole moments. This enables the parametrization of charges and LJ parameters using a simple linear scaling of the existing force fields parameters. We are interested in extending this methodology to derive force fields of highly polar molecules, such as formamide, and in particular reproduce the liquid dielectric constant. The latter property is an important input parameter in theories that model the solvent as a dielectric continuum, which have applications on solubility computations.

In this work we will test the applicability of the procedure introduced by Salas et al. [9] to derive a new force field that describes the properties of liquid formamide. We will build on the work of Caleman et al. in the web page http : //www.virtualchemistry.org and reparametrize the OPLS/AA force field. Advancing the discussion below we will show that the linear scaling of the force field parameters is not enough to derive an accurate force field of formamide. This can be explained as a consequence of the strong hydrogen bonding between molecules, which depends very strongly on the magnitude of the atomic charges. We present alternative strategies to fit the atomic charges, which rely on quantum mechanical computations and the introduction of polarization contributions, which allow us to successfully derive an accurate force field. Further, we test the transferability of our formamide force field to investigate liquid binary mixtures, in particular formamide/hexan-2one. This mixture is an example of two components with a highly different polar behavior.

The paper is organized as follows. We firstly describe in Section 2 the simulation details. A discussion of the different optimization procedures employed in this work follows. We then discuss our results and close the paper with a summary of the main conclusions and final remarks.

\section{$2 \quad$ Force field and simulation details}

We employ here a generic potential based on the OPLS/AA force field, which combines intramolecular terms (bond stretching, angle bending and torsion) with intermolecular and intramolecular non-bonded interactions [20]. The OPLS/AA functional form for the intermolecular contributions contains Lennard-Jones and coulombic terms,

$$
V\left(r_{i j}\right)=\left\{4 \varepsilon_{i j}\left[\left(\frac{\sigma_{i j}}{r_{i j}}\right)^{12}-\left(\frac{\sigma_{i j}}{r_{i j}}\right)^{6}\right]+\frac{q_{i} q_{j}}{4 \pi \varepsilon_{0} r_{i j}}\right\} f_{i j}
$$

where $r_{i j}$ is the distance between atoms $i$ and $j, q_{i}$ is the partial charge on atom $i, \varepsilon_{0}$ is the vacuum permittivity, $\sigma_{i j}$ and $\epsilon_{i j}$ are the effective atom diameter and interaction strength, respectively. The factor $f_{i j}$ is 0.5 for interactions involving 
intramolecular interactions between atom pairs separated by three or more bonds, and 1 for intermolecular interactions [3]. The cross interactions are calculated using the geometric mixing rules: $\sigma_{i j}=\left(\sigma_{i i} \sigma_{j j}\right)^{1 / 2}$ and $\epsilon_{i j}=\left(\epsilon_{i i} \epsilon_{j j}\right)^{1 / 2}$.

Molecular dynamics simulations in the isothermal-isobaric ensemble, NPT, with isotropic fluctuations of volume, were performed to compute the liquid density and dielectric constant at the standard pressure, 1 bar. These simulations involved typically 500 molecules.

Simulations in the canonical ensemble, NVT, were also performed to compute the surface tension of the liquid, by simulating an explicit liquid-vapor interface containing, typically, 1000 molecules. The interface was generated by setting up a liquid slab surrounded by vacuum in a simulation box with periodic boundary conditions in the three spatial directions. The dimensions of the simulation cell were $L_{x}=L_{y}=52 \AA$ with $L_{z}=3 L_{x}$, where $z$ being the normal direction to the liquid-vapor interface.

The GROMACS 4.5.4 package [21] was employed in all the simulations presented in this work. The equations of motion were solved using the leap-frog algorithm with a time step of 2 fs. The temperature was coupled to the Nosé-Hoover thermostat with a parameter $\tau_{T}=0.2 \mathrm{ps}$ while the pressure was coupled to the Parrinello-Rahman barostat with a coupling parameter $\tau_{P}=0.5 \mathrm{ps}$. The bond distances in the formamide molecule were kept rigid by using the LINCS algorithm[22] and the molecule was considered to be planar. The electrostatic interactions were computed with the Particle Mesh Ewald approach [23] with a tolerance of $10^{-6}$ for the real space contribution, with a grid spacing of $1.2 \AA$ and spline interpolation of order 4 . In the isotropic NPT simulations the real part of the Ewald summation and the LJ interactions were truncated at $12 \AA$. Long range corrections for the LJ energy and pressure were added. The dielectric constant was computed using the dipole moment fluctuations of the system [24].

The density and dielectric constant were calculated in the same simulation for at least 120 ns after an equilibration period of 10 ns. For the surface tension computations in the NVT ensemble the cutoff was set to $25 \AA$, since the surface tension depends on the truncation of the interactions [25, 17] and the interface cross sectional area [26, 27]. The equilibration period for the interfacial simulations was 2 ns and the results for the average properties were obtained over an additional 6 ns trajectory. The equations used to calculate the dielectric constant, surface tension, self-diffusion coefficient and pair distribution function are given as Supplementary Information.

The quantum mechanical computations were performed with the Gaussian09 set of programs[28] using the M062X functional [29] and the $6-311++\mathrm{g}^{* *}$ basis set [30, 31]. The hybrid functional was chosen because it was parametrized with molecules containing nitrogen and the basis set represents better the atomic orbitals.

Amongst other approaches, we derived the atomic charges from populations anal- 
yses that rely on the fitting of the molecular electrostatic potential using both the CHELPG [32] and Merz-Singh-Kollman (MK)[33, 34] schemes. The criteria for convergence of these approaches is based on energy, density or orbital gradient [35].

\section{Results}

First, ab initio calculations and molecular dynamics simulations were performed to obtain the optimum set of partial charges and the LJ parameters to determine dielectric constant, surface tension and liquid density in good agreement with experimental data. At the end, the original and new force field of formamide were used to study a binary mixture between hexan-2-one and formamide to analyze their miscibility properties.

\subsection{Linear Scaling approach}

Linear scaling has been successfully employed in the past to compute the atomic charges of weakly polar molecules [9]. The method can be applied by perturbing the parameters of existing force fields, and re-scaling the parameters to reproduce specific thermodynamic properties. We tested the accuracy of the OPLS/AA force field in determining the dielectric constant of formamide by performing NPT computer simulations at $298.15 \mathrm{~K}$ and 1 bar. In agreement with previous studies [16] the dielectric constant, 50, is much lower than the experimental result, 109. Hence, as a first step to correct the force field we applied the method introduced by Salas et al. [9]. We re-scale linearly the charges only, while the rest of the force field parameters were the same as in the OPLS/AA force field. The liquid density, dielectric constant and self-diffusion coefficient are reported in Table 1. The molecular dipole moment and liquid density increase by increasing the atomic charge but the dielectric constant features a small dependence with charge, except for very large charges. We find that $\epsilon$ increases about 5 units $(\sim 10 \%)$ for a $20 \%$ increase in the charge. Interestingly, further increase in the charge, $35 \%$, results in a very large reduction of the dielectric constant, from 55 to 11 . We have tested the convergence of our calculations by computing the average of the dipole moment, $<\mathbf{M}>$ (where $\mathbf{M}=\sum_{\mathbf{i}=\mathbf{1}}^{\mathbf{N}} \mathbf{q}_{\mathbf{i}} \mathbf{r}_{\mathbf{i}}$ ) of a system containing $N$ particles and $\mathbf{r}_{i}$ the position of atom $i$. For the equilibrium situation the average of the dipole moment magnitude, $\langle M\rangle$ must converge to zero. We checked (see $\langle M\rangle$ in Table 1) that our simulations were well converged. Hence the strong non-linear effects observed in the dielectric constant must be connected to a strong modification of the liquid upon increasing the atomic charges. In particular, the non-linear dependence of $\epsilon$ with the atomic charge indicates that orientational correlations are important in determining the dielectric response of formamide. The reduction of $\epsilon$ at large charges is connected to a strong modification of the hydrogen 
bond interactions $(\mathrm{C}-\mathrm{H} \ldots \mathrm{O}$ and $\mathrm{N}-\mathrm{H} . . . \mathrm{O})$ in the liquid. The electrostatic interactions between the hydrogen bonding sites leads to an enhancement of the liquid structure, with formation of molecular clusters and a concomitant reduction of the liquid dynamics, which is reflected in a smaller diffusion coefficient at higher atomic charges (see Table 1).

\begin{tabular}{|ccccc|}
\hline Atom $/ f_{q}$ & 1.0 & 1.1 & 1.2 & 1.35 \\
\hline $\mathrm{C}$ & 0.500 & 0.550 & 0.600 & 0.675 \\
$\mathrm{O}$ & -0.500 & -0.550 & -0.600 & -0.675 \\
$\mathrm{HC}$ & 0.000 & 0.000 & 0.000 & 0.000 \\
$\mathrm{~N}$ & -0.760 & -0.836 & -0.912 & -1.026 \\
$\mathrm{H}$ & 0.380 & 0.418 & 0.456 & 0.513 \\
$\mathrm{H}$ & 0.380 & 0.418 & 0.456 & 0.513 \\
\hline \hline$\mu$ & 4.20 & 4.62 & 5.04 & 5.68 \\
$\rho$ & 1122.6 & 1152.2 & 1178.4 & 1202.1 \\
$\epsilon$ & 50.2 & 55.1 & 55.1 & 11.2 \\
$<M$ & 1.32 & 2.23 & 6.07 & 110.82 \\
$\mathrm{D} / 10^{-5}$ & 0.51 & 0.14 & 0.013 & - \\
\hline
\end{tabular}

Table 1: Atomic charges (in units of the electron charge) for the original OPLS/AA force field. The charge scaling factor is $f_{q}$. The molecular dipole moment in Debyes is $\mu$. The liquid density in $\mathrm{kg} / \mathrm{m}^{3}$ is $\rho$, the average dipole moment of the system in Debyes is $\langle M\rangle$ and the self diffusion-coefficient in $\mathrm{cm}^{2} / \mathrm{s}$ is $\mathrm{D}$.

\subsection{Atomic charges via ab initio computations}

We have shown in the previous section that it is not possible to reproduce the experimental dielectric constant by simply re-scaling the atomic charges of the OPLS/AA force field. We consider in the following ab initio approaches to fulfill this task. We note that several approaches can be used at this respect. In this work we consider computations in vacuum as well as additional computations introducing polarization effects. As noted in section 2 our computations were performed using density functional theory (DFT) with the M062X [29] functional and the 6-311++g** basis set $[30,31]$. We have considered approaches based on population analyses, and fitting to the molecular electrostatic potential (CHELPG and MK), as well as wave function analyses: Mulliken [36] and natural bond orbital (NBO) [37, 38]. We have collected all our results in Table 2 for isolated molecules in vacuum. The bond distances and angles were obtained in this work with ab initio calculations and they were essentially equal to those of the original OPLS/AA. These charges in combination with the rest of all original OPLS/AA parameters, including the bond distances and angles, were used to perform NPT molecular dynamics computations of the dielectric constant. 


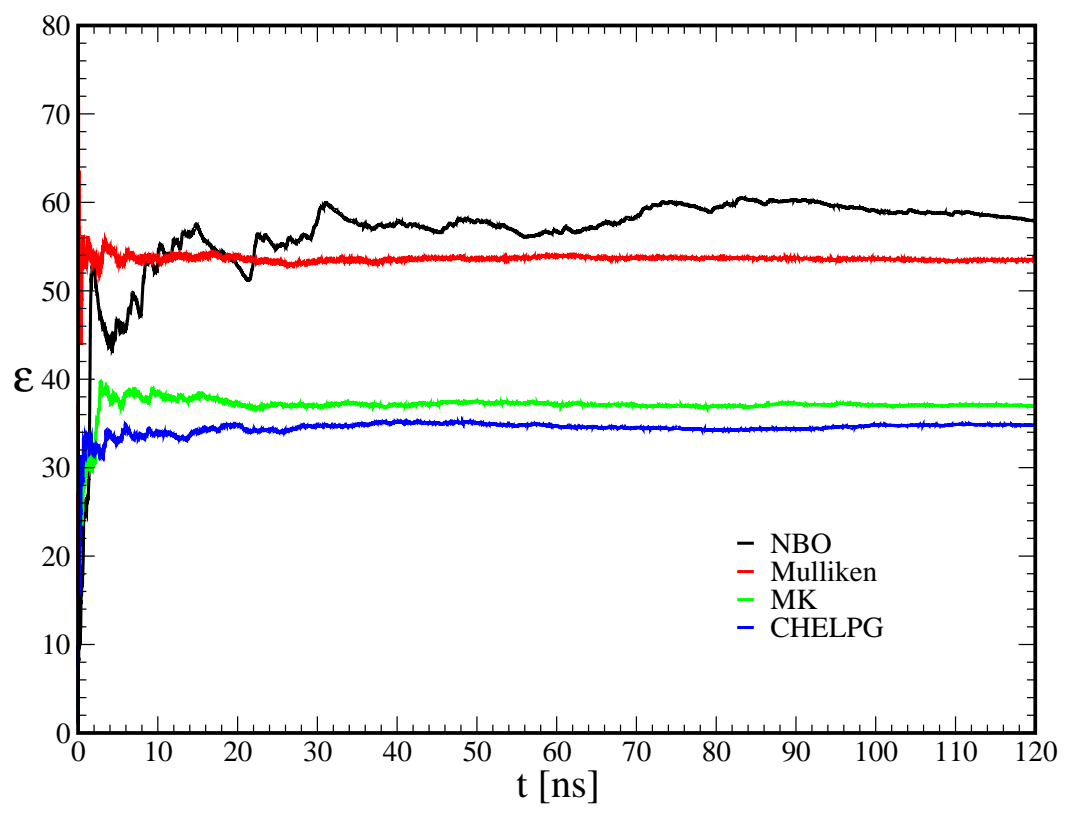

Figure 1: Results of the dielectric constant of formamide at $298.15 \mathrm{~K}$ and 1 bar from ab initio calculation on isolated molecules in vacuum using the Mulliken, NBO, MK and CHELPG approaches.

The values for the average dipole moment (see Table 2) indicate that our computations were well converged. This can also be seen in the running average of the dielectric constant (see Figure 1), which reaches the average value in 20-40 ns.

Different atomic charge electrostatic schemes (Mulliken, CHELPG and MK) gave similar dipole moments but overestimated the experimental result, $3.73 \mathrm{D}$, in the gas phase [39]. Among the standard methods to obtain charges and moments, it has been found that the MK results show an appreciable dependence on the orientation of the molecule [40]. The dielectric constant for the atomic charges obtained with the different electrostatic methods are in poor agreement with experiment. In particular the CHELPG and MK charge distribution lead to fairly low dielectric constant. Again the differences observed in the dielectric constant do not correlate with the dipole moment values. As a matter of fact the Mulliken and MK approaches predict the same dipole moment, $4.02 \mathrm{D}$, while their dielectric constants are very different, 53.5 and 35.9 respectively. This is again an indication that orientation correlations play a significant role in determining the dielectric constant. Further the MK atomic charges in the atoms that mediate hydrogen bonds (N, O) are much higher than in the Mulliken case.

The main conclusion from the analysis above is that the new ab initio charge distributions do not improve the description of the dielectric constant. The poor performance of these charge distributions prompted us to consider polarization ef- 


\begin{tabular}{|ccccc|}
\hline Atom & Mulliken [36] & NBO [37, 38] & CHELPG [32] & MK [33, 34] \\
\hline $\mathrm{C}$ & 0.1203 & 0.5464 & 0.7267 & 0.6553 \\
$\mathrm{O}$ & -0.3798 & -0.6097 & -0.5835 & -0.5554 \\
$\mathrm{HC}$ & 0.1035 & 0.1045 & 0.0197 & 0.0128 \\
$\mathrm{~N}$ & -0.3919 & -0.8346 & -0.9407 & -0.9623 \\
$\mathrm{H}$ & 0.2852 & 0.4000 & 0.4277 & 0.4423 \\
$\mathrm{H}$ & 0.2628 & 0.3934 & 0.3895 & 0.4073 \\
\hline \hline$\mu$ & 4.02 & 4.85 & 4.04 & 4.02 \\
$\rho$ & 1094.7 & 1194.6 & 1146.8 & 1149.6 \\
$<M$ & 1.5 & 10.5 & 0.8 & 2.2 \\
$\epsilon$ & 53.5 & 57.5 & 34.8 & 35.9 \\
$\mathrm{D} / 10^{-5}$ & 1.20 & 0.04 & 0.28 & 0.30 \\
\hline
\end{tabular}

Table 2: The atomic charges derived from different population analyses for isolated molecules in vacuum: Mulliken, NBO, CHELPG and MK. The variables $\mu, \rho,\langle M\rangle, \epsilon$ and $\mathrm{D}$ have the same meaning and units reported in Table 1. All data correspond to the thermodynamic state, $298.15 \mathrm{~K}$ and 1 bar.

fects in our ab initio computations. These polarization effects are important in many chemical and biochemical processes in solution. Existing continuum models, e.g. the polarizable continuum model, takes into account the influence of the solvent through an effective dielectric constant [41, 42, 43]. The solvation model density (SMD) by Marenich et al. [44] provides another alternative to handle these effects. That model is able to approximate the interaction of a molecule with a solvent. We performed electronic structure computations including polarization effects. The SMD approach was combined with the M062X functional and the same basis sets employed before $\left(6-311++\mathrm{g}^{* *}\right)$. The atomic charges were derived by using the population analysis methods mentioned above. We compile all the results from those theories in Table 3. We found that the inclusion of the polarization effects through the SMD method results increased in a $\sim 30 \%$ the molecular dipole moment irrespective of the method employed for the population analysis. The bond distances and angles were also obtained in this work using the $a b$ initio calculations with polarizable effects and they were identical to the original OPLS/AA values. The new results were compared in Table 4 with experimental data and with the original OPLS/AA values. The molecular geometry was not affected because the inclusion of solvent effects, therefore, the change in the molecular dipole moment is due only to the charge distribution. To obtain the dielectric constant and other properties, we performed NPT MD simulations of liquid formamide using the new charge distribution with all the original OPLS/AA parameters including bond distances and angles. The density increases slightly, $\sim 10 \%$, with respect to the data reported in Table 2, while the dielectric constant varies significantly depending on the method used to derive the atomic 
A

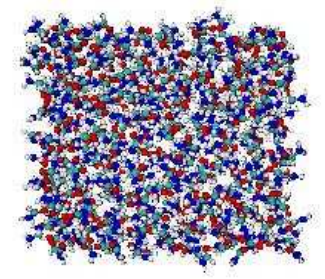

$\mathrm{C}$

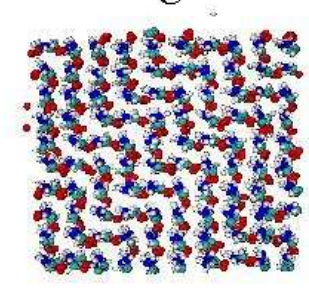

B

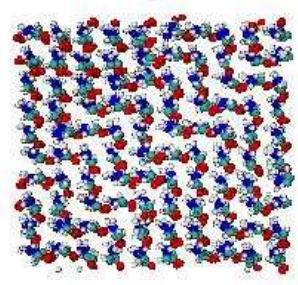

D

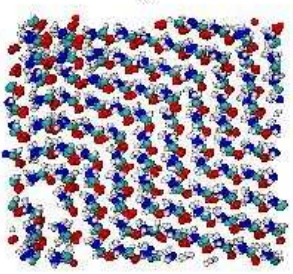

Figure 2: Typical snapshots of formamide systems simulated with charges derived from different approaches with polarizable effects. A) Mulliken, B) NBO, C) CHELPG and D) MK.

charges. The dielectric constant obtained from the Mulliken analysis, 132.5, is of the order, but higher than the experimental value of 109. A snapshot for the last configuration is shown in Fig. 2-A where a liquid configuration is observed. We found that the simulations with the other atomic charges (NBO, CHELPG, MK) featured in general poor stability, with large fluctuations in the simulation cell dipole moment, which precluded the computation of reliable values for the dielectric constant. The highly negative and positive charges on the oxygen/nitrogen and carbon lead to strong ordering effects. Inspection of simulation snapshots are shown from Fig. 2-B to Fig. 2-D. It is remarkable the strong impact of the atomic charges in driving the formation of solid structures with the molecules arranging themselves in well defined rows. Figure 3 shows typical the oxygen-oxygen and nitrogen-nitrogen pair distribution functions where it is observed a liquid structure for results with the Mulliken method and a solid-like structure for the NBO, CHELPG and MK approaches. The radial distribution functions for oxygen-nitrogen and carbon-carbon are provided as Supplementary Information.

\subsection{Derivation of an accurate force field for liquid formamide}

We have shown that the direct application of standard ab initio approaches to fit the atomic charges does not provide a route to obtain an accurate force field to simulate 


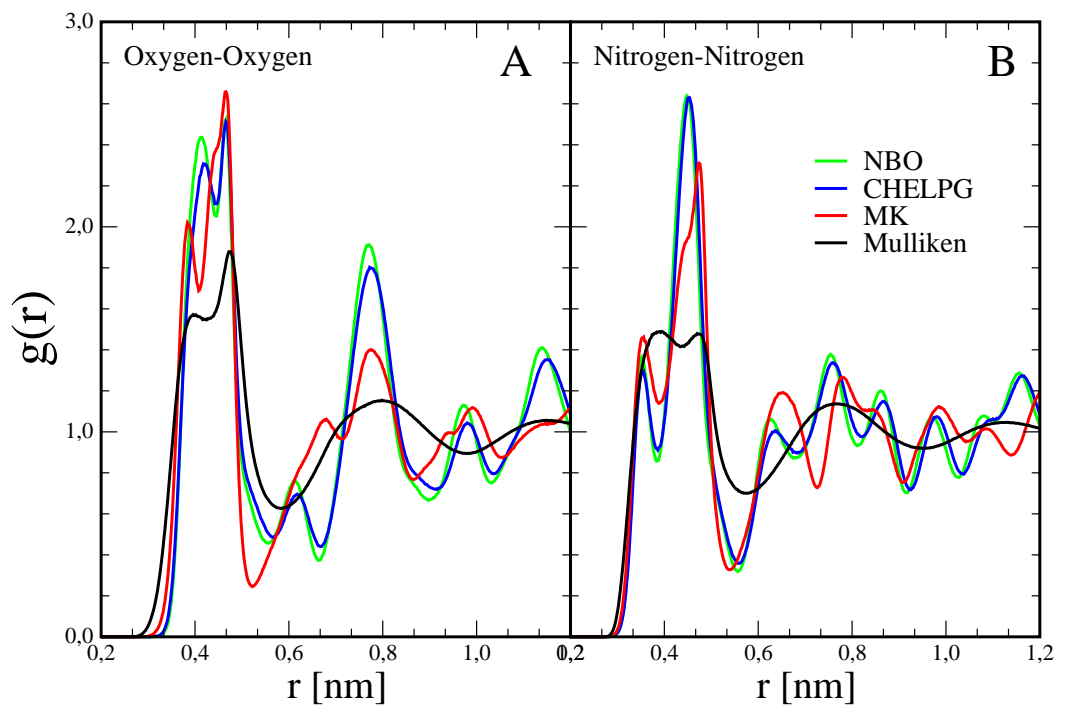

Figure 3: Radial distribution functions for formamide. A) oxygen-oxygen B) nitrogennitrogen. The results were obtained with charges derived from different approaches including polarizable effects.

the liquid phase of formamide. Hence, we considered the approach reported by Salas et al. [9]. Firstly, we derived the atomic charges from the Mulliken analysis reported in the previous section in combination with the SMD approach. The charges were then optimized to match the experimental dielectric constant using molecular dynamics simulations, keeping constant the rest of the original OPLS/AA parameters. Then, we kept the optimal charges fixed and performed a linear re-scaling of the OPLS/AA Lennard-Jones values for $\epsilon_{L J}$ to obtain the experimental surface tension. Finally, the original $\sigma_{L J}$ OPLS/AA values were also linearly re-scaled to obtain the experimental liquid density keeping constant the new charges and new $\epsilon_{L J}$ parameters.

In order to quantify the impact of the new fitted parameters on the properties of the liquid we define a ratio that measures the relative percent change (RPC) of the new parameter with respect to the original OPLS/AA one. For instance, the relative change for the charges is given by, $R P C=\left(q / q_{0}-1\right) \times 100$, where $q_{0}$ is the OPLS/AA charge. Similar equations were employed for the $\epsilon_{L J}$ and $\sigma_{L J}$ parameters. To facilitate the comparison of the results obtained with different parameter sets, we normalized the target properties, $\epsilon, \gamma$ and $\rho$, by their corresponding experimental value. We show in Figure 4 the variation of the normalized property with the relative percent change. The largest changes in the properties are observed upon modifying the atomic charges and the interaction strength, $\epsilon_{L J}$. From the three properties investigated the bulk density features the smallest change, while the surface tension and dielectric constant both are more sensitive to changes in the charges and interaction strength.

To check whether a result is acceptable, it is necessary to define a tolerance. To 


\begin{tabular}{|ccccc|}
\hline Atom & Mulliken & NBO & CHELPG & MK \\
\hline $\mathrm{C}$ & 0.1472 & 0.5562 & 0.7804 & 0.7079 \\
$\mathrm{O}$ & -0.5561 & -0.7537 & -0.7709 & -0.7450 \\
$\mathrm{HC}$ & 0.1845 & 0.1471 & 0.0178 & 0.0494 \\
$\mathrm{~N}$ & -0.4382 & -0.8031 & -0.9202 & -0.9296 \\
$\mathrm{H}$ & 0.3341 & 0.4232 & 0.4614 & 0.4724 \\
$\mathrm{H}$ & 0.3285 & 0.4303 & 0.4315 & 0.4449 \\
\hline \hline$\mu$ & 5.71 & 6.33 & 5.80 & 5.83 \\
$\rho$ & 1202.1 & 1334.2 & 1244.2 & 1283.3 \\
$\langle M$ & 5.1 & 1718.0 & 1516.8 & 1821.2 \\
$\epsilon$ & 132.5 & - & - & - \\
\hline
\end{tabular}

Table 3: Atomic charges obtained from different population analyses. The polarization effects were taken into account through the SMD model. [44]. The properties reported in the table have the same meaning and units as those in Tables 1 and 2. All the simulations were performed in the NPT ensemble at $298.15 \mathrm{~K}$ and 1 bar.

\begin{tabular}{cccc}
\hline Property & Experiment[45, 46] & Original & New \\
\hline Bond distance & & & \\
C - N & 1.368 & 1.335 & 1.333 \\
N - H & 1.027 & 1.010 & 1.012 \\
C - O & 1.212 & 1.229 & 1.229 \\
C - HC & 1.125 & 1.090 & 1.098 \\
Bond angle & & & \\
N-C-O & 125.0 & 122.9 & 124.6 \\
N-C-HC & 112.7 & 114.0 & 113.8 \\
C-N-H & 118.7 & 119.8 & 119.1 \\
C-N-H & 119.7 & 120.0 & 120.6 \\
O-C-HC & 122.5 & 123.0 & 120.4
\end{tabular}

Table 4: Bond distances in $\AA$ and bond angles in degrees for formamide obtained in this work, labeled New, with ab initio calculations using polarization effects SMD and M062X/6-311g $++\mathrm{g}^{* *}$ levels of theory. The experimental and the original values for the OPLS/AA are also given.

do this we define the relative error $\Delta X=\left|\left(X_{\text {exp }}-X_{M D}\right)\right| \times 100 / X_{\text {exp }}$, where $M D$ and exp denote simulation and experimental results, respectively. We set $8 \%, 5 \%$ and $1 \%$ as acceptable relative errors for $\epsilon, \gamma$ and $\rho$, respectively.

We show in Figure 4-A the impact of re-scaling only the magnitude of the atomic charges obtained through the Mulliken analysis. This force field overestimates the value of the three properties investigated here: density $(7 \%)$, dielectric constant $(22 \%)$, and surface tension $(50 \%)$ (see data at $\mathrm{RPC}=0)$. These properties can be 

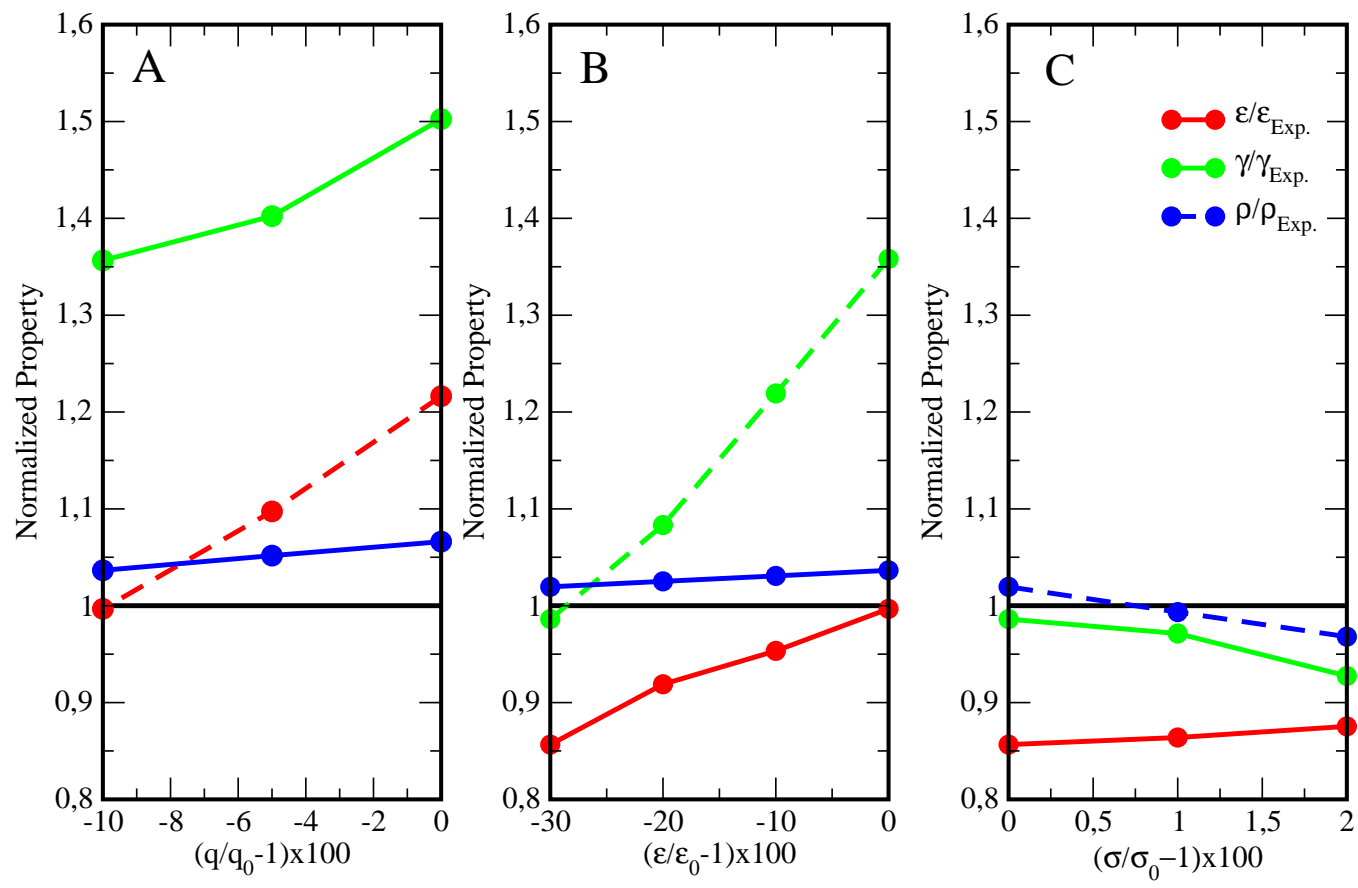

Figure 4: Variation of the normalized properties: dielectric constant, surface tension, and bulk density with the relative change in the force field parameters. Black lines correspond to experimental values. The lines with filled circles correspond to: dielectric constant (red), surface tension (green) and liquid density (blue). A) Scaling of the charges. B) Scaling of $\epsilon_{L J}$ and C) Scaling of $\sigma_{L J}$. The dashed lines indicate the property that has been optimized in each panel. Dielectric constant in panel A, surface tension in panel B and density in panel C. The experimental values were: $\epsilon_{E x p}=108.94, \gamma_{E x p}=57.03 \mathrm{mN} / \mathrm{m}$ and $\rho_{E x p}=1129.0$ $\mathrm{kg} / \mathrm{m}^{3}$

brought into closer agreement with the experimental data by re-scaling the original charges towards smaller values. In this way it is possible to obtain accurate result for the dielectric constant, improving at the same time the surface tension (see Figure 4-A). But getting better agreement for the latter requires a reparametrization of the interaction strength. This has a knock on effect on the dielectric constant, which features now a 15\% underestimation (see Figure 4-B). Finally, we considered the density. We find that only a small change of $1 \%$ in $\sigma_{L J}$ is needed to reproduce the experimental density. After this final fitting, both the density and the surface tension (see Figure 4-C) are, within our required tolerance, equal to the experimental values. However, we found that the the simulated dielectric constant is $13 \%$ lower than the the experimental one. To resolve this problem and reduce the difference between simulation and experiment, we performed a second re-scaling of all the parameters, starting with the values shown in Figure. 4 -C and $\left.\left(\sigma / \sigma_{0}-1\right) \times 100\right)=1$. This new iteration involved a $5 \%$ re-scaling of the atomic charges and $0.5 \%$ for $\sigma_{L J}$. The $\epsilon_{L J}$ 
values were not modified. The results obtained with those values are not shown in Fig. 4. The final optimized force field parameters are given in Table 5, and compared with the original OPLS/AA parameters. We have collected in Table 6 the simulated and experimental data for the three target properties discussed above. The data show that the new force field provides a good description of the dielectric constant, surface tension and liquid density. We have included in the Supporting information the numerical data of the liquid density, dielectric constant and surface tension for all the systems investigated in this work.

\begin{tabular}{|cccc|}
\hline Original & $\mathrm{q}(\mathrm{e})$ & $\sigma(\mathrm{nm})$ & $\varepsilon(\mathrm{kJ} / \mathrm{mol})$ \\
\hline $\mathrm{C}$ & 0.5000 & 0.37500 & 0.43932 \\
$\mathrm{O}$ & -0.5000 & 0.29600 & 0.87864 \\
$\mathrm{HC}$ & 0.0000 & 0.24200 & 0.06276 \\
$\mathrm{~N}$ & -0.7600 & 0.32500 & 0.71128 \\
$\mathrm{H}$ & 0.3800 & 0.00000 & 0.00000 \\
$\mathrm{H}$ & 0.3800 & 0.00000 & 0.00000 \\
\hline $\mathrm{New}$ & $\mathrm{q}(\mathrm{e})$ & $\sigma(\mathrm{nm})$ & $\varepsilon(\mathrm{kJ} / \mathrm{mol})$ \\
\hline $\mathrm{C}$ & 0.1398 & 0.380625 & 0.307524 \\
$\mathrm{O}$ & -0.5283 & 0.300440 & 0.615048 \\
$\mathrm{HC}$ & 0.1753 & 0.245630 & 0.043932 \\
$\mathrm{~N}$ & -0.4163 & 0.329875 & 0.497896 \\
$\mathrm{H}$ & 0.3174 & 0.000000 & 0.000000 \\
$\mathrm{H}$ & 0.3121 & 0.000000 & 0.000000 \\
\hline
\end{tabular}

Table 5: Original and new parameters obtained in this work for the OPLS/AA force field.

\begin{tabular}{|cccc|}
\hline Property & Original & New & Experiment[47],[48] \\
\hline$\epsilon$ & 50.2 & 107.6 & 108.9 \\
$\gamma$ & 61 & 57.0 & 57.0 \\
$\rho$ & 1122 & 1128.5 & 1129.0 \\
\hline
\end{tabular}

Table 6: Results obtained in this work using the OPLS/AA force field with the original and new parameters obtained in this work. The calculated relative errors using the new parameters are: $0.44 \%, 1.14 \%$ and $0.41 \%$ for $\epsilon, \gamma$ and $\rho$, respectively. The surface tension is given in $\mathrm{mN} / \mathrm{m}$, and density in $\mathrm{kg} / \mathrm{m}^{3}$.

An important element of a force field are the combination rules employed to computer cross interactions. These rules determine the transferability of the potential to simulate, e.g. mixtures. The OPLS/AA force field uses the geometric mixing rule, while other force fields, such as the general Amber force field (GAFF), use the socalled Lorentz-Berthelot where $\sigma_{i j}=\left(\sigma_{i i}+\sigma_{j j}\right) / 2$. The combination rule for $\epsilon_{i j}$ is the same in both force fields. To test these different implementations of the combining 
rules we run additional simulations to compute the target properties at $298.15 \mathrm{~K}$. The liquid calculations were carried out at 1 bar. The factor $f_{i j}$ appearing in equation (1) was identical in both mixing rules, 0.5 for intramolecular interactions between atoms separated by three or more bonds and 1.0 for atoms in two different molecules. The parameters obtained in this work are used in both calculations. The results are shown in Table 7. The combination rule is shown to have a small impact on the liquid and interfacial properties, with a maximum deviation in the surface tension of $\sim 5 \%$. Generally, the observed differences are within our target tolerance. Considering the small differences between both combination rules and because we want to compare results from the OPLS/AA force field, we will be using the geometric rule to perform the rest of the computations presented in this work.

\begin{tabular}{|cccc|}
\hline Property & Geometric & Lorentz-Berthelot & Experiment \\
\hline$\epsilon$ & 107.6 & 105.7 & 108.9 \\
\hline$\gamma$ & 57.0 & 54.4 & 57.0 \\
\hline$\rho$ & 1128.5 & 1117.3 & 1129.0 \\
\hline
\end{tabular}

Table 7: Results for the target properties using the new parameters but with two mixing rules for $\sigma i j$. The surface tension is given in $\mathrm{mN} / \mathrm{m}$ and the density in $\mathrm{kg} / \mathrm{m}^{3}$.

In order to check the accuracy of our force field and the general improvement over the existing OPLS/AA potential we performed additional NPT and NVT computations of liquid formamide as a function of the temperature. The new force field parameters reproduce the experimental target properties as a function of temperature within the request tolerance in the available temperature range, see Fig. 5. It has to be noted that formamide is not stable for temperatures above $373 \mathrm{~K}$ as it decomposes to hydrogen cyanide and water [49]. Overall, the new parametrization provides an improvement on all the target properties over the existing OPLS/AA force field. The relative error for the (original, new) parameters with respect experimental data are $(54,1)$ for the dielectric constant, $(-7,0)$ for the surface tension and $(0.6,0)$ for the liquid density. We have obtained a force field that reproduces the dielectric constant keeping at the same time the accuracy in predicting density and surface tension.

\subsection{Testing the transferability of the new force field: the hexan-2-one/formamide binary mixture}

In order to test the transferability of our new force field parameters we studied a liquid binary mixture consisting of formamide and hexan-2-one. As noted in the introduction, this mixture is a stringent test as it involves two polar liquid with significantly different dielectric constants. Further, hexan-2-one is a ketone used in industrial applications as general solvent and in paints, with a low dielectric constant 


\begin{tabular}{|cccc|}
\hline Property & Temperature & This work & Experiment[47],[48] \\
\hline$\epsilon$ & 298.15 & 107.6 & 108.94 \\
& 308.15 & 103.2 & 104.91 \\
& 318.15 & 99.6 & 100.94 \\
& 328.15 & 94.9 & 97.04 \\
\hline$\gamma$ & 298.15 & 57.7 & 57.0 \\
& 323.15 & 55.5 & 54.9 \\
& 348.15 & 53.5 & 52.8 \\
& 373.15 & 51.2 & 50.7 \\
\hline$\rho$ & 298.15 & 1128.5 & 1129.0 \\
& 308.15 & 1120.7 & 1120.5 \\
& 318.15 & 1113.3 & 1111.9 \\
& 328.15 & 1105.4 & 1103.3 \\
\hline
\end{tabular}

Table 8: Results of dielectric constant, surface tension $(\mathrm{mN} / \mathrm{m})$ and liquid density $\left(\mathrm{kg} / \mathrm{m}^{3}\right)$ of formamide as a function of temperature $(\mathrm{K})$ obtained with the new parameters for OPLS/AA.

(15) at $298.15 \mathrm{~K}$, and liquid density of $807 \mathrm{~kg} / \mathrm{m}^{3}$ [16]. It is known that this molecule has low solubility in water, $14 \mathrm{~g} / \mathrm{L}$ [50] while formamide shows a much higher solubility in water, namely, $1000 \mathrm{~g} / \mathrm{L}$ [49]. Hence, it is expected that a mixture with both components will present a low miscibility.

First, a liquid simulations with 256 hexan-2-one was performed at 298.15 and 1 bar obtaining a liquid density of $821 \mathrm{~kg} / \mathrm{m}^{3}$ and dielectric constant of 10.5 in acceptable agreement with experimental and simulation data. [16]

To analyze and compare the accuracy of the original and new parameters derived here for formamide, we performed simulations of the mixture. Two pre-equilibrated configurations of formamide and hexan-2-one were placed side by side in an elongated simulation cell such that the two liquids were initially completely demixed. In one simulation we employed the original OPLS/AA parameters for both components, while in a second independent run we used the original parameters for hexan-2-one and the new OPLS/AA parameters for formamide. The cross interactions were computed with the geometric combining rules. Long simulations involving $250 \mathrm{~ns}$ of equilibration and up to $2.3 \mu$ s of production time were performed to ensure a proper relaxation of the system. We computed the density profile of every component for different sub-averages to follow the miscibility. The results for the first simulation averaged over the last $25 \mathrm{~ns}$ are shown in Figure 6-A. We find that the original OPLS/AA predicts complete miscibility between both liquids. For the second simulation, which was performed with the new formamide potential derived in this work we find that the two liquids form a well defined liquid-liquid interface (see Figure 6$\mathrm{B})$, hence clearly showing that they are inmiscible, a result that is expected in view 

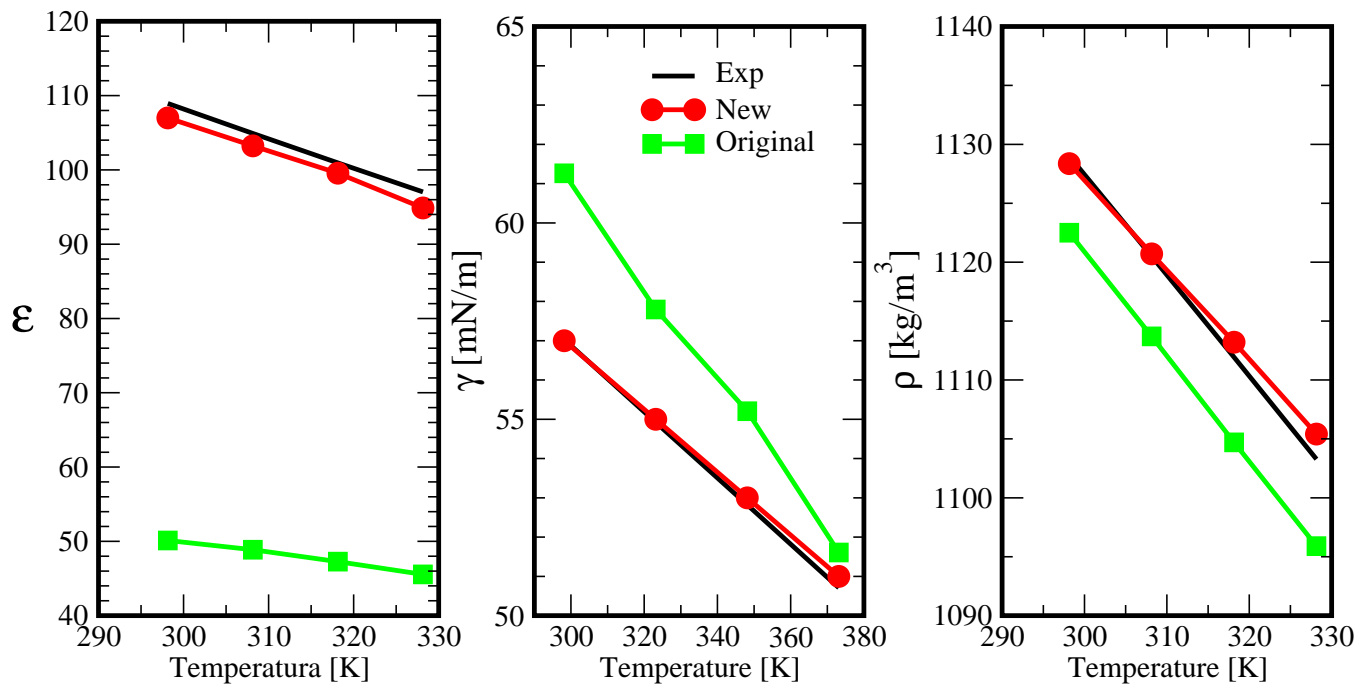

Figure 5: Results of target properties for formamide as a function of temperature using the OPLS/AA force field. A) Dielectric constant, B) Surface tension and C) liquid density. The results for the original and new parameters are shown with green squares and red circles, respectively. The experimental values are shown with continuous lines.

of the large difference in dielectric constant. We were unable to find experimental values of solubility for that mixture. There is a large amount of liquid for hexan-2-one and formamide to evaluate the composition of every component in each liquid phase. The hexan-2-one is more soluble in formamide than formamide in hexan-2-one.

In order to understand the role of molecular interactions in the solubility of the mixtures studied in the previous section, the free energy of solvation of a hexan-2-one molecule in a liquid formamide was obtained using the thermodynamic integration, TI, method [51]. The change in free energy, $\Delta G$, is obtained using that method over a dummy variable, $\lambda$, which couples to the Hamiltonian, and that enables the systematic modification of the latter from a given reference system, for which the free energy may be known,

$$
\Delta G=\int_{0}^{1}\left\langle\frac{\partial H(\lambda)}{\partial \lambda}\right\rangle d \lambda
$$

where $\Delta G$ is the free energy of solvation and $H$ is the Hamiltonian. As the coupling parameter, $\lambda$, changes from " 0 " to " 1 " the hamiltonian interpolates between two different systems, so that it is possible to switch off the interactions, and hence compute the work (chemical potential) required to insert or extract a molecule in a fluid under NPT conditions. Practically, obtaining accurate free energy differences between two 

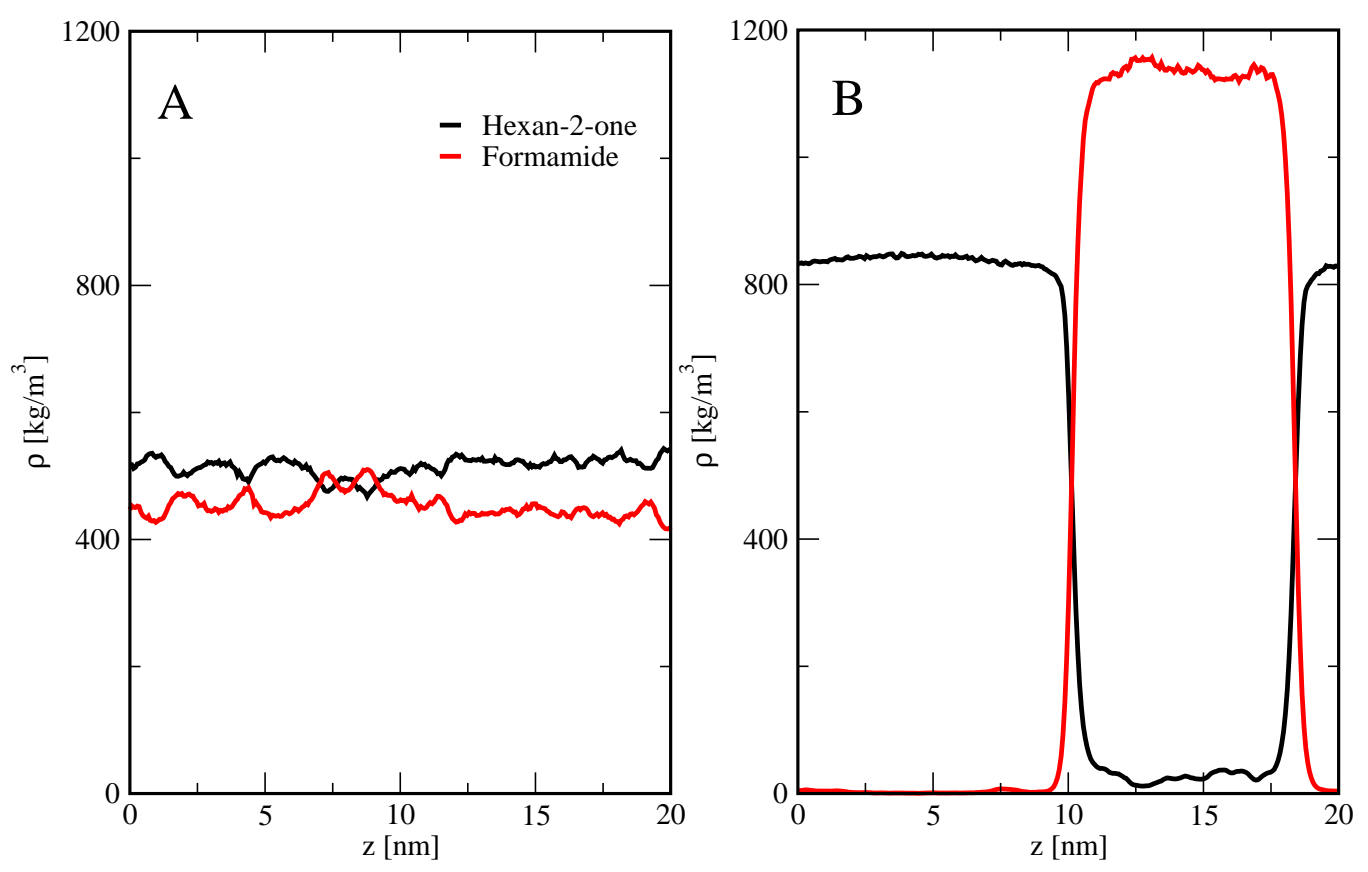

Figure 6: Density profiles of the hexan-2-one/formamide mixture. A) Simulations with the original OPLS/AA parameters for both components. B) Simulations with the original OPLS/AA parameters for hexan-2-one and with the new parameters derived in this work for formamide.

states, requires small changes of $\lambda$. Further it has been shown that better convergence is obtained when the different interactions (coulombic and dispersion) are switched off independently. It is customary to switch off the coulombic interaction first $[52,53]$. The simulations were performed in the NPT ensemble at $298 \mathrm{~K}$ and 1 bar pressure. We then computed, using the perturbation method outlined above, the solvation free energy of one molecule of hexan-2-one in a liquid formamide. The results of free energy change as a function of $\lambda$ for the hexa-2-one/formamide using the original parameters for both components and for the hexa-2-one/formamide using the new parameters for formamide are shown in a figure in the Supplementary Information. This computation should be taken as an approximation to the true free energy of solvation, as unlike in the free energy computation, the hexa-2-one molecule is not at infinite dilution in the formamide phase, although the concentration is rather low, see Fig. 6. The solvation free energy of the mixture with the original parameters for both components were $8.28 \mathrm{~kJ} / \mathrm{mol}$ and $14.56 \mathrm{~kJ} / \mathrm{mol}$ when switching off the Lennard-Jones and coulombic interactions, respectively, so that, the total change in free energy is $\Delta G=22.84 \mathrm{~kJ} / \mathrm{mol}$. When the new parameters are used, the solvation free energy were $2.75 \mathrm{~kJ} / \mathrm{mol}$ and $13.21 \mathrm{~kJ} / \mathrm{mol}$ when switching off the Lennard-Jones and coulombic interactions, respectively. In this case $\Delta G=15.96 \mathrm{~kJ} / \mathrm{mol}$. It is im- 
portant to note that the difference in free energy between both simulations is $9 \%$ for the coulombic interactions and $81 \%$ for the LJ contribution. The energy to insert a molecule of hexan-2-one in formamide is the negative of the values given above. The results show, that the attraction between both molecules in the insertion process is larger in the first case than in the second. Those calculations suggest that the phase separation is not due to coulombic interactions or to a large difference in dielectric constant of two components ( $\Delta \gamma=100$ from simulations of this work) but that the miscibility is driven by the short ranged interactions. The free energy provided insights about the importance of the charge distribution in describing the solubility.

\section{Conclusions}

We have presented a systematic procedure that combines ab initio and molecular dynamics computations, that allows the derivation of optimum parameters for the formamide force field. The approach targets three different properties; the dielectric constant, liquid density and surface tension. These properties are fitten by systematically varying (via linear re-scaling), the atomic charges, and the interaction strength ( $\left.\epsilon_{L J}\right)$ and atomic diameters $\left(\sigma_{L J}\right)$ appearing in the Lennard-Jones potential used to describe the dispersion interactions. First the charges from quantum calculations were fitted to reproduce the dielectric constant. Second, the Lennard-Jones parameter $\epsilon_{L J}$ were fitted to match the surface tension at the vapor-liquid interface and finally a third step the $\sigma_{L J}$ parameters were fitted to reproduce the liquid density. Two cycles were needed to find the optimum parameters. The idea behind the method is that a change in a specific force field parameter affects more one property than the others. The parameters can then be fitted independently if appropriate target properties are chosen. For instance, changes in the $\epsilon_{L J}$ affect much more the surface tension than dielectric constant and liquid density. Starting from the widely used OPLS/AA force field we derived new parameters that provide a good prediction of liquid density, surface tension and crucially the dielectric constant of liquid formamide. The new model represent a significant improvement in the prediction of this quantity, which is now in very good agreement with the experiment. The previous OPLS/AA estimate underestimated this property by over $50 \%$. Although the parameters were fitted at $300 \mathrm{~K}$, they show good transferability in the prediction of the liquid properties at higher temperatures. We did also analyze the impact of the combination rules in the accuracy of the force field. We find that the results are fairly independent on whether the geometric or Lorentz-Berthelot combination rules are employed to derive cross interactions between dissimilar atoms.

We performed additional simulations of a liquid-liquid mixture, consisting of hexan-2-one and formamide. This mixture provides a further benchmark to test the transferability of our force field parameters. We have shown that the miscibility 
behaviour is strongly dependent on the force field used. The original OPLS/AA parameters predict full miscibility, wile the new force field for formamide predicts that the two liquids are inmiscible. The free energy calculations suggest that the driven force of solubility comes from the short ranged interactions. The large difference in dielectric constant between hexan-2-one and formamide, $\Delta \epsilon=100$ for the calculated values in this work, does not seem to be the reason for which the systems do not mix. More work is needed to have definite conclusions on this subject. Nevertheless, reproducing the dielectric constant allows to obtain the optimum Lennard-Jones parameters if the surface tension and liquid density are chosen as target properties.

The results from this work also show that polarizable models are not needed to obtain the dielectric constant and surface tension of highly polar fluids and to describe the miscibility of mixtures.

\section{Supporting Information}

Definitions and numerical results of thermodynamic, structural and electric properties, selected pair distributions for the systems studied in this work and the free energy change as a function of $\lambda$. This material is available free of charge via the Internet at http://pubs.acs.org.

\section{Acknowledgments}

G.A.M.M., E.N.R. and J.A. thank Laboratorio de Supercómputo for allocation time. F.B. would like thank the EPSRC (EP/J003859/1) and The Research Council of Norway (Project 221675) for financial support.

\section{References}

[1] Puhovski, U. P.; Rode, B. M. Structure and dynamics of liquid formamide. Chem. Phys. 1995, 190, 61-82.

[2] Kritsana, P.; Reinhart, A. A test particle model potential for formamide and molecular dynamics simulations of the liquid. J. Chem. Phys. 1987,86, 51175145.

[3] Jorgensen, W. L.; Maxwell, D. S.; Tirado-Rives, J. Development and all-atom Force Field on Conformational Energetics and Properties of Organic Liquid. J. Am. Chem. Soc. 1996, 118, 11225-11236. 
[4] Wang, J.; Wolf, R. M.; Caldwell, J. W.; Kollman, P. A.; Case, D. A. Development and Testing of a General Amber Force Field. J. Comput. Chem. 2004, 25, 11571174 .

[5] Jorgensen, W. L.; Swenson, C. J. Optimized Intermolecular Potential Functions for Amides and Peptides. Structure and Properties of Liquid Amides. J. Am. Chem. Soc. 1985, 107, 569-578.

[6] van Leeuwen, M. E.; Smit, B. J. Molecular Simulation of the Vapor-Liquid Coexistence Curve of Methanol. J. Phys. Chem. 1995, 99, 1831-1833.

[7] Wick, C. D.; Stubbs, J. M.; Rai, N.; Siepmann, J. I. Transferable Potentials for Phase Equilibria. 7. Primary, Secondary, and Tertiary Amines, Nitroalkanes and Nitrobenzene, Nitriles, Amides, Pyridine, and Pyrimidine J. Phys. Chem. B. 2005, 109, 18974-18982.

[8] Nath, S. K.; A, Escobedo, F. A.; de Pablo, J. On the simulation of vapor-liquid equilibria for alkanes. Journal of Chemical Physics. 1998, 108, 9905-9911.

[9] F. J. Salas, G. A. Méndez-Maldonado, E. Núñez-Rojas, G. E. Aguilar-Pineda, H. Domínguez $^{\mathrm{c}}$ and J. Alejandre, Just accepted, J. Chem. Theory Comput, 2014.

[10] Xie, W; Pu, J.; Mackerell, A. D.; Gao, J. Development of a Polarizable Intermolecular Potential Function (PIPF) for Liquid Amides and Alcanes. J. Chem. Theory Comput. 2007, 3, 1878-1889.

[11] Essex, J. W.; Jorgensen, W. L. Dielectric constants of formamide and dimethylformamide via computer simulation. J. Phys. Chem. 1995, 99, 17956-17962.

[12] Puhovski, Y. P.; Safonova, L. P.; Rode, B. M. Molecular dynamic simulations of a liquid formamide and $\mathrm{N}, \mathrm{N}$-dimethylformamide with new quantum mechanical potential. J. Molec. Liq. 2003, 103-104, 15-31.

[13] Cordeiro, J. M. M. C(Single Bond)H(Dotted Bond)O and N(Single Bond)H(Dotted Bond)O hydrogen bonds in liquid amides investigated by Monte Carlo simulation. Int. J. Quantum Chem. 1997. 65, 709 -717.

[14] Bako, I; Megyes, T.; Bálint, S.; Chihaia, V.; Bellissent-Funel M. C.; Krienke, H.; Kopf A.; Suh, S. H. Hydrogen bonded network properties in liquid formamide. J. Chem. Phys. 2010. 132, 014506.

[15] Harder, E.; Anisimov, V. M.; Whitfield, T.; MacKerell A.D. Jr; Roux B. Understanding the Dielectric Properties of Liquid Amides from a Polarizable Force Field. J. Phys. Chem. B. 2008. 112, 3509-3521. 
[16] Caleman, C.; van Maaren, P.J.; Hong, M.; Hub, J. S.; Costa, L.T.; van der Spoel, D. Force Field Benchmark of Organic Liquids: Density, Enthalpy of Vaporization, Heat Capacities, Surface Tension, Isothermal Compressibility, Volumetric Expansion Coefficient, and Dielectric Constant. J. Chem. Theory Comput. 2012, 8, 61-74.

[17] Zubillaga, R. A.; Labastida, A.; Cruz, B.; Martínez, J. C.; Sánchez, E.; Alejandre, J. Surface Tension of Organic Liquids Using the OPLS/AA Force Field. J. Chem. Theory Comput. 2013, 9, 1611-1615.

[18] Alejandre, J.; Chapela, G. A.; Saint-Martin, H.; Mendoza, N. A non-polarizable model of water that yields the dielectric constant and the density anomalies of the liquid: TIP4Q. Phys. Chem. Chem. Phys. 2011, 13, 19728-19740.

[19] Fuentes-Azcatl, R.; Alejandre, J. Non-Polarizable Force Field of Water Based on the Dielectric Constant: TIP4P/E. J. Phys. Chem. B. 2014, 118 1263-1272.

[20] Jorgensen, W.L.; Tirado-Rives J. The OPLS [optimized potentials for liquid simulations] potential functions for proteins, energy minimizations for crystals of cyclic peptides and crambin. J. Am. Chem. Soc. 1988, 110, 1657-1666.

[21] Hess, B.; Kutzner, C.; van der Spoel, D.; Lindahl, E. GROMACS 4: Algorithms for highly efficient, load-balanced, and scalable molecular simulation. J. Chem. Theory. Comput. 2008, 4, 435-447.

[22] Hess, B.; Bekker, H.; Berendsen, H. J. C.; Fraaije, J. G. E. M, LINCS: A linear constraint solver for molecular simulations, J. Comp. Chem, 1997, 18, 14631472 .

[23] Essmann, U; Perera, L.; Berkowitz, M. L.; Darden, T.; Lee, H.; Pedersen L. G. A smooth particle mesh Ewald method. J. Chem. Phys. 1995, 103, 8577-8592.

[24] J.P. Hansen and I.R. McDonald, Theory of Simple Liquids, 2013, $4^{t} h$ edition, Elsevier Ltd.

[25] Truckymchuk, A.; Alejandre, J. Computer simulations of liquid/vapor interface in Lennard-Jones fluids: Some questions and answers J. Chem. Phys. 1999, 111, 8510-8523.

[26] Orea, P.; López-Lemus, J.; Alejandre J. Oscillatory surface tension due to finitesize effects. J. Chem. Phys. 2005, 123, 114702-114813.

[27] Gonzalez-Melchor, M.; Bresme, F.; Alejandre, J. Molecular dynamics simulations of the surface tension of ionic liquids. J. Chem. Phys. 2005, 122, 104710. 
[28] Gaussian 09, Revision B.01, M. J. Frisch, G. W. Trucks, H. B. Schlegel, G. E. Scuseria, M. A. Robb, J. R. Cheeseman, G. Scalmani, V. Barone, B. Mennucci, G. A. Petersson, H. Nakatsuji, M. Caricato, X. Li, H. P. Hratchian, A. F. Izmaylov, J. Bloino, G. Zheng, J. L. Sonnenberg, M. Hada, M. Ehara, K. Toyota, R. Fukuda, J. Hasegawa, M. Ishida, T. Nakajima, Y. Honda, O. Kitao, H. Nakai, T. Vreven, J. A. Montgomery, Jr., J. E. Peralta, F. Ogliaro, M. Bearpark, J. J. Heyd, E. Brothers, K. N. Kudin, V. N. Staroverov, T. Keith, R. Kobayashi, J. Normand, K. Raghavachari, A. Rendell, J. C. Burant, S. S. Iyengar, J. Tomasi, M. Cossi, N. Rega, J. M. Millam, M. Klene, J. E. Knox, J. B. Cross, V. Bakken, C. Adamo, J. Jaramillo, R. Gomperts, R. E. Stratmann, O. Yazyev, A. J. Austin, R. Cammi, C. Pomelli, J. W. Ochterski, R. L. Martin, K. Morokuma, V. G. Zakrzewski, G. A. Voth, P. Salvador, J. J. Dannenberg, S. Dapprich, A. D. Daniels, O. Farkas, J. B. Foresman, J. V. Ortiz, J. Cioslowski, and D. J. Fox, Gaussian, Inc., Wallingford CT, 2010.

[29] Zhao, Y.;Truhlar D. G. The M06 suite of density functionals for main group thermochemistry, thermochemical kinetics, noncovalent interactions, excited states, and transition elements: two new functionals and systematic testing of four M06-class functionals and 12 other functionals. Theor. Chem. Acc. 2008, 120, 215-241.

[30] McLean, A. D.; Chandler, G. S. Contracted Gaussian-basis sets for molecular calculations. 1. 2nd row atoms, $\mathrm{Z}=11-18$. J. Chem. Phys. 1980, 72, 5639-5648.

[31] Raghavachari, K.; Binkley, J. S; Seeger, R; Pople, J. A. Self-Consistent Molecular Orbital Methods. 20. Basis set for correlated wave-functions. J. Chem. Phys. 1980, 72, 650-654.

[32] Breneman, C .M; Wiberg, K. B. Determining atom-centered monopoles from molecular electrostatic potentials. The need for high sampling density in formamide conformational analysis. J. Comp. Chem. 1990, 11, 361-373.

[33] Singh U. C.; Kollman, P. A. An approach to computing electrostatic charges for molecules. J. Comp. Chem. 1984, 5, 129-45.

[34] Besler, B. H.; Merz Jr, K. M.; Kollman P. A. Atomic charges derived from semiempirical methods. J. Comp. Chem. 1990, 11, 431-439.

[35] H. B. Schlegel and J. J. McDouall, in Computational Advances in Organic Chemistry, Ed. C. Ögretir and I. G. Csizmadia (Kluwer Academic, The Netherlands, 1991) $167-85$.

[36] Mulliken, R. S. Electronic Population Analysis on LCAO-MO Molecular Wave Functions. J. Chem. Phys. 1955, 23, 1833. 
[37] Foster, J. P.; Weinhold, F. Natural hybrid orbitals. J. Am. Chem. Soc. 1980, 102, $7211-7218$.

[38] Reed, A. E.; Weinhold, F. Natural bond orbital analysis of near-Hartree-Fock water dimer. J. Chem. Phys. 1983, 78, 4066-73.

[39] Nelson Jr, D. R.; Lide, D. R.; Maryott, A. A. Selected Values of electric dipole moments for molecules in the gas phase. National Standart Reference Data System, 1967.

[40] Sigfridsson, E.; Ryde, U. Comparison of Methods for Deriving Atomic Charges from the Electrostatic Potential and Moments, J. Comput. Chem, 1998, 19, $377-395$

[41] Miertus̃, S; Scrocco, E.; Tomasi, J. Electrostatic interaction of a solute with a continuum. A direct utilizaion of $a b$ initio molecular potentials for the prevision of solvent effects. Chem. Phys. 1981, 55, 117-129.

[42] Miertus̃, S; Tomasi, J. Approximate evaluations of the electrostatic free energy and internal energy changes in solution processes. Chem. Phys. 1982, 65, 239-45.

[43] Pascual-Ahuir, J. L.; Silla E.; Tuñon, I. GEPOL: An improved description of molecular surfaces. III. A new algorithm for the computation of a solventexcluding surface. J. Comp. Chem. 1994, 15, 1127-38.

[44] Marenich, A. V.; Cramer C. J.; Truhlar, D. G. Universal Solvation Model Based on Solute Electron Density and on a Continuum Model of the Solvent Defined by the Bulk Dielectric Constant and Atomic Surface Tensions. J. Phys. Chem. B. 2009, 113, 6378-96.

[45] Costain C.C. and Dowling J.M., J. Chem. Phys. 32,158-187, 1960).

[46] Kitano M. and Kichutsu K., Chem. Soc. Japan,47, 67-76, 1974.

[47] Lide D.R., CRC Handbook of Chemistry and Physics 90th edition, CRC Press: Cleveland, Ohio (2009).

[48] Yaws C.L, Thermophysical properties of chemicals and hydrocarbons, William Andrew, April, 2088.

[49] Kirk-Othmer. Encyclopedia of Chemical Technology, 3rd ed.; John Wiley and sons. New York, NY, 1980.

[50] Flick, E. W. Industrial Solvents Handbook, 3rd ed.; Noyes Publications: Park Ridge, N J, 1985. 
[51] Villa, A.; Mark, A. E. Calculation of the Free Energy of solvation for neutral analogs of amino acids side chains. J. Comput. Chem. 2002, 23, 548-553.

[52] Pohorille, A.; Jarzynski, C.; Chipot, C. Good Practices in Free-Energy Calculations. J. Phys. Chem. B 2010, 114, 10235-10253.

[53] Christ, C. D.; Mark, A. E.; van Gunsteren, W. F. Basic Ingredients of Free Energy Calculations: A Review. J. Comput. Chem. 2009, 31, 1569-1582. 